\title{
A distinct cytokine and chemokine profile at the genital mucosa is associated with HIV-1 protection among HIV-exposed seronegative commercial sex workers
}

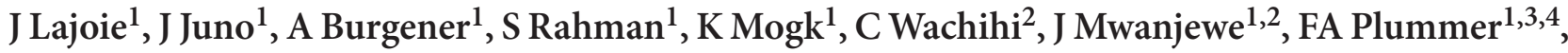 \\ $\mathrm{J} \mathrm{Kimani}^{1,2}$, TB Ball ${ }^{1,3,5}$ and KR Fowke ${ }^{1,4,5}$
}

The predominance of HIV-1 sexual transmission requires a greater understanding of the interaction between HIV-1 and the mucosal immune system. The study of HIV-1-exposed seronegative (HESN) individuals serves as a model to identify the correlates of protection and to aid in microbicide development. A total of 22 cytokines/chemokines were analyzed at the systemic and mucosal compartments in 57 HESN, 51 HIV-1-negative, and 67 HIV-1-infected commercial sex workers from Nairobi, Kenya. HESN individuals had significantly lower expression of monokine induced by interferon- $\gamma$ (MIG), interferon- $\gamma$-induced protein 10 (IP-10), and interleukin-1 $\alpha$ (IL-1 $\alpha$ ) in their genital mucosa compared with controls. HESN cytokine expression also distinctly correlates with mucosal antiproteases, suggesting that HESN individuals have a unique pattern of mucosal chemokine/cytokine expression, which may result in reduced trafficking at the mucosa. These data support the immune quiescence model of protection, whereby lower T-cell activation/recruitment at the mucosal compartment reduces HIV-1 target cell numbers and is an important component of natural protection from HIV-1.

\section{INTRODUCTION}

The UNAIDS (Joint United Nations Programme on HIV and AIDS) estimates that more than half of all people living with HIV/AIDS are women. ${ }^{1}$ It is estimated that, in Africa, women are twice as likely to contract HIV-1 during vaginal intercourse compared with men and are thus considered to be a vulnerable population. ${ }^{1}$ The development of a female microbicide that provides protection against HIV-1 acquisition is a promising preventative strategy, and a recent trial of a $1 \%$ vaginal gel formulation of Tenofovir was able to reduce the risk of HIV-1 acquisition by up to $54 \%$ among women with high adherence. ${ }^{2}$ However, other recent clinical trial such as the $0.5 \% \mathrm{PRO} 2000$ has failed to prevent HIV infection. ${ }^{3}$ Microbicide efficacy therefore remains low and developing an effective and protective formulation is still a critical priority in the fight against HIV. Given that evidence from both HIV and Simian immunodeficiency virus infection studies suggests a more substantial impact of the virus on the mucosal immune system than its systemic counterpart, perturbation of mucosal immunity, combined with the predominance of mucosal transmission in the HIV epidemic, makes the study of mucosal immunology a critical focus in the HIV field. A better understanding of the earliest effects of HIV infection on mucosal immunity and of the potential mechanisms of natural immunity against HIV infection is required for the development of an effective preventative strategy.

Over the years, many factors have been associated with both susceptibility to, and protection from, HIV-1 infection, ${ }^{4-8}$ but some of the most interesting findings have emerged from the description of HIV-exposed seronegative (HESN) individuals. ${ }^{9}$ Despite repeated exposure, these individuals show a natural resistance to HIV-1 and have been identified in various cohorts across the world. ${ }^{10-15}$ The study of these cohorts has led to the identification of multiple factors correlated with protection

${ }^{1}$ Department of Medical Microbiology, University of Manitoba, Winnipeg, Manitoba, Canada. ${ }^{2}$ Kenyan AIDS Control Program, Nairobi, Kenya. ${ }^{3}$ Public Health Agency of Canada, Winnipeg, Manitoba, Canada. ${ }^{4}$ Department of Community Health Sciences, University of Manitoba, Winnipeg, Manitoba, Canada. ${ }^{5}$ Department of Medical Microbiology, University of Nairobi, Nairobi, Kenya. Correspondence: KR Fowke (fowkekr@cc.umanitoba.ca) 
from HIV-1 acquisition (reviewed in ref. 16). The Pumwani Sex Worker Cohort in Nairobi, Kenya, includes a group of HESN women $(n=150)$ who experience high mucosal exposure to HIV-1 infection but remain seronegative for $>7$ years of follow-up. ${ }^{10}$ Those women represent an extreme phenotype of the HESN individuals and are considered to be relatively resistant to HIV-1 infection. ${ }^{10}$ Many studies have examined the immunological and genetic correlates of protection among these women and have demonstrated the presence of systemic HIV-specific T-cell responses, ${ }^{17-19}$ polymorphisms in the IRF1 (interferon regulatory factor 1 ) gene, and increased mucosal levels of antiproteases. ${ }^{20,21}$ These factors support a model of immune quiescence among Pumwani HESNs where decreased levels of T-cell activation and host proteins required for HIV-1 replication limit available target cells. ${ }^{22}$ A detailed understanding of how immune quiescence may be manifested at the female genital tract (FGT), however, is still lacking. Factors that affect lymphocyte trafficking to the FGT and that regulate the inflammatory state of the mucosa could be key contributors to the induction of immune quiescence at the genital mucosa.

In HIV-1 infection, expression of certain chemokines has been associated with HIV susceptibility and disease progression. In cervicovaginal lavage (CVL) samples, susceptibility to infection has been previously associated with high expression of interleukin (IL) $-8,{ }^{23}$ whereas a high level of RANTES (regulated upon activation, normal T-cell expressed, and secreted), ${ }^{24}$ secretory leukocyte protease inhibitor, ${ }^{25}$ and interferon- $\alpha(\text { IFN }-\alpha)^{26}$ have been associated with protection from HIV-1 infection. Following establishment of infection, monocyte chemotactic protein-1 (MCP-1) has been shown to positively correlate with viral load. ${ }^{27}$ Higher systemic levels of monokine induced by interferon- $\gamma$ (MIG) and interferon- $\gamma$ induced protein (IP)-10 have been observed in HIV-positive commercial sex workers (CSWs) from Benin compared with HIV-1-uninfected CSWs. Although the impact of chemokine expression both mucosally and systemically on HIV-1 infection and progression is clear, the pattern of expression among HESN individuals of the Pumwani cohort has not previously been described.

The aim of this study was, therefore, to examine the profile of pro- and anti-inflammatory cytokines and chemokines involved in immune activation and lymphocyte recruitment in the CVL of CSWs from the Pumwani cohort. Here, we report that HESN individuals express lower CVL levels of IL- $1 \alpha$ and two IFN- $\gamma$-inducible chemokines, MIG and IP-10, compared with the levels observed in HIV-1-negative CSWs ( $<3$ years in cohort) and HIV-1-infected CSWs. The HIV-1-negative CSW group represents a group of HIV-1-susceptible CSWs with similar sexual risk behaviors to HESN individuals and is considered as our baseline control group. Inclusion of HIV-1infected CSWs in this study serves to demonstrate the impact of HIV-1 infection at the mucosal surface. Our data support the existence of the immune quiescence phenotype at the genital mucosa and may suggest improved strategies for microbicide development.

\section{RESULTS}

\section{Sociodemographic information and sexually transmitted infections among study groups}

Sociodemographic and sexually transmitted infection data were collected to address confounding variables that may affect acquisition of HIV-1 and the mucosal immune response (Table 1). Because of the criteria involved in the definition of the length of follow-up required of HESN women, there are significant differences in the age and duration of sex work between the three groups $(P<0.0001)$, which was controlled for during subsequent analysis. The number of reported clients per week, the number of regular partners, and condom usage were equivalent between the three groups. The prevalence of Chlamydia trachomatis and/or Neisseria gonorrhoeae infection and bacterial vaginosis was similar between groups.

Table 1 Sociodemographic, behavioral, and clinical factors among the study cohort

\begin{tabular}{|c|c|c|c|c|}
\hline & $\begin{array}{l}\text { HESN CSW } \\
(N=57)\end{array}$ & $\begin{array}{c}\text { HIV-1-negative CSW } \\
(N=51)\end{array}$ & $\begin{array}{l}\text { HIV-1-infected CSW } \\
(N=67)\end{array}$ & $P$-value \\
\hline Age & $43.6(8.5)$ & $33.1(6.9)$ & $39.9(7.8)$ & $<0.0001^{a, b}$ \\
\hline Clients per week & $17.5(17.2)$ & $19.1(16.2)$ & $17.7(21.7)$ & NS \\
\hline Number of regular partner & $1.6(2.3)$ & $2.0(2.3)$ & $1.5(2.1)$ & NS \\
\hline CD4 count & NA & NA & $431.8(303.9)$ & NA \\
\hline
\end{tabular}

CSW, commercial sex worker; CT, Chlamydia trachomatis; GC, Neisseria gonorrhoeae; HESN, HIV-exposed seronegative; NA, nonapplicable; NS, nonsignificant. All risks factors were collected by a questionnaire. Gynecological exams and biological sampling were performed by a physician. For age, duration of sex work, clients per week, number of regular partner, and CD4 count, data in the table are presented as mean \pm s.d. in brackets. For CT/GN infection and Bacterial vaginosis, data in the table indicated the number of infection/number of all samples tested (percentage of infection).

$P$-values for comparison across all groups were calculated with one-way analysis of variance (Kruskal-Wallis test) for the age, duration of sex work, clients per week, and number of regular partner. The $\chi^{2}$ test for the CT/GN and Bacterial vaginosis. For difference between two groups, Dunn's multiple comparison tests were used.

aDifference observed between HESN CSW and HIV-1-negative CSW, $P<0.0001$

${ }^{b}$ Difference observed between new negative CSW and HIV-1-positive CSW, $P<0.0001$. 


\section{Cytokine and chemokine expression in the CVL}

No significant differences were observed between the three groups for the expression of macrophage inflammatory protein (MIP)-3 $\alpha$, MIP-1 $\alpha$, MIP-1 $\beta$, interferon-inducible T-cell $\alpha$-chemoattractant (I-TAC), fractalkine, IFN- $\alpha 2$, IFN- $\gamma$, IL-1 $\beta$, IL-1r $\alpha$, IL-2, IL-7, IL-10, IL-15, IL-17, MCP-1 and MCP-3, the macrophage-derived chemokine, sCD40L, sIL-2R $\alpha$, and tumor necrosis factor- $\alpha$ in the CVL (Supplementary Table S1 online). The HIV-1-negative CSWs exhibited higher expression of IL-6 compared with both the HESN and HIV-1-infected CSWs (Kruskal-Wallis test, $P=0.04$; post-tests nonsignificant). Notably, compared with the HIV-1-negative and HIV-1-infected groups, the HESN group exhibited lower expression of IL-1 $\alpha$ (Kruskal-Wallis test, $P=0.04$; post-test $<0.05$ for both), MIG (Kruskal-Wallis test, $P=0.013$; post-test $<0.05$ for both), and IP-10 (Kruskal-Wallis test, $P=0.015$; post-test $P<0.05$ for both; Figure 1). Both MIG and IP-10 are IFN- $\gamma$-inducible chemokines that bind the cellular receptor CXCR3. Although not reaching statistical significance, the expression of IL-8 trended toward being lower in the HESN group (Kruskal-Wallis test, $P=0.08)$.

Within the HESN group, considerable variation in chemokine/cytokine levels was evident. Although no correlation between chemokine/cytokine levels and sociodemographic/ clinical factors was observed (data not shown), other factors such as the menstrual cycle, routine douching, and type of douching product used could contribute to the observed variation.

\section{Cytokine and chemokine expression in the plasma}

In general, whereas HIV immunology studies primarily focus on peripheral blood sample, previous reports have observed differences in cytokine/chemokine expression patterns between the mucosal and systemic environments, demonstrating that the observations made in the systemic compartment cannot be extrapolated to the mucosa. Leukocytes will migrate from the periphery to mucosal tissues according to a chemokine gradient, and the study of these two different compartments can give further insight into possible leukocyte trafficking. Here, the expression of the same panel of chemokines/cytokines was analyzed in matched plasma samples (where available) to characterize the mucosal/peripheral gradient as well as the difference between the two compartments (Supplementary Table S2 online). Interestingly, the systemic patterns of differential cytokine/chemokine expression did not necessarily mirror those detected in the CVL. Although the HESN group tended to exhibit lower levels of CVL cytokines compared with the other study groups, the significant systemic differences predominately showed a higher expression of cytokines/chemokines in the HIV-1-infected group (Figure 2).

When we compared the mucosal expression of the chemokines/cytokines to the corresponding systemic concentrations, we observed a different pattern of expression between the two compartments. In each group, the expressions of IL-1 $\alpha$, IL-1 $\beta$, IL-1r $\alpha$, IL-6, IL-7, and IL-8 were higher in the mucosal compartment compared with the systemic compartment.
Comparatively, the expressions of I-TAC, fractalkine, macrophage-derived chemokine, sCD40L, and tumor necrosis factor- $\alpha$ were higher in the systemic compartment compared with the mucosal compartment (Figure 3 and Supplementary Table S3 online).

Interestingly, significant differences in the expression gradients were also present between the three CSW groups. IL-2 and MCP-3 were higher in the systemic compartment compared with the CVL in the HIV-1-negative CSWs and HIV-1-infected CSW s but not in HESN individuals. The HIV-1-negative and HESN groups showed an elevation of IL-15 and IFN- $\alpha 2$ in the mucosal compartment that was not observed in the HIV-1-infected CSWs. Most interestingly, the HIV-1-negative CSWs showed higher expression of MIG in the mucosal vs. systemic compartment, whereas the HESN group exhibited the opposite gradient. Furthermore, the HESN CSWs was the only group to show the presence of a gradient for IP-10, which was higher in the systemic compartment compared with the mucosal compartment (Figure 3 and Supplementary Table S3 online).

\section{Cervical mononuclear cell (CMC) analysis}

Because lower levels of MIG and IP-10 at the mucosa might be compensated for by increased expression of CXCR3 + on lymphocytes at the FGT, we assessed CXCR3 expression on cervical mononuclear CD $4+$ and CD8 + T cells, as well as expression of the activation marker human leukocyte antigen (HLA)-DR (Figure 4). We observed no significant difference in the relative proportions of CD $4+$ or CD8 + T cells between the three groups of CSWs. Despite lower levels of MIG and IP-10 in the CVL of the HESN population, there was no difference in the proportion of expression or median fluorescence intensity of CXCR3 on CD4 + or CD8 + T cells. The HESN group did trend toward lower expression of HLA$\mathrm{DR}$ on $\mathrm{CD} 8+\mathrm{T}$ cells (relative proportion $P=0.07$, median fluorescence intensity $P=0.1$ ).

\section{Correlation between chemokine/cytokine and antiprotease expression at the FGT}

One of the roles of chemokine/cytokine expression at the FGT is to control the state of immune activation, but other factors such as antiprotease play an important part in that control and can regulate chemokine/cytokine expression. Antiproteases function to counter inflammation, and their expression has previously been associated with HIV-1 protection in the Pumwani cohort. ${ }^{28}$ For that reason, we examined the correlation between the chemokine/cytokine detected in this study and the expression of five mucosal antiproteases among matched CVL samples. Two major observations arose from this analysis. First, the cytokines/chemokines reduced among HESN individuals (MIG, IP-10, and IL-1 $\alpha$ ) exhibited different patterns of correlation with antiproteases between the study groups. MIG positively correlated with cystatin A, A2ML1, and serpin B4 expression in the HESN group $(P=0.01, P=0.01$, and $P=0.004$, respectively), but not in the other study groups. IP-10 positively correlated with A2ML1 only in the HESN group $(P=0.01)$. 

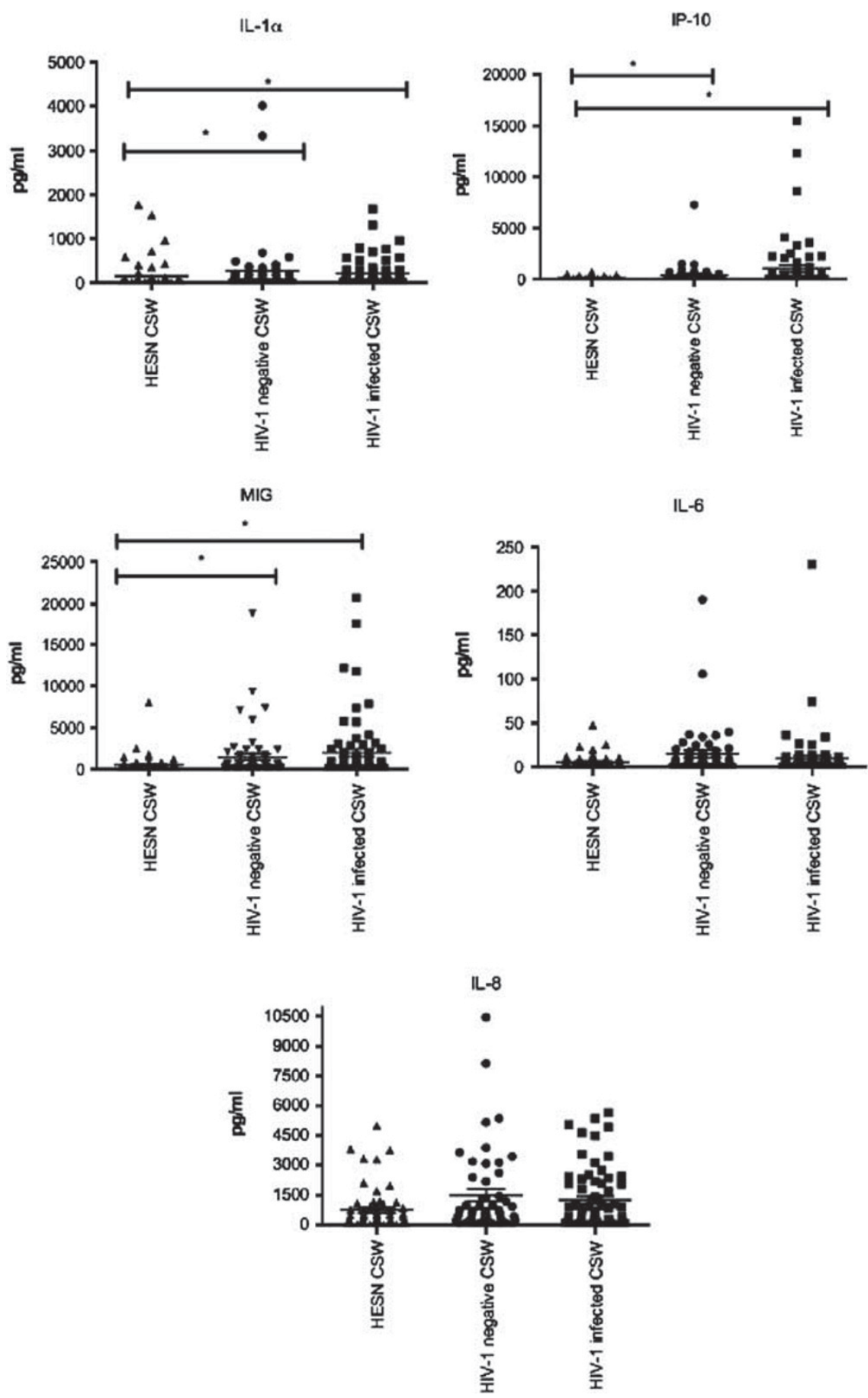

Figure 1 Distribution of the significant association. Cervicovaginal lavage (CVL) cytokine and chemokine levels were analyzed with Milliplex MAP multiplex and normalized with standard curves. Sample measurements below the lower detection limit were assigned a value of half the lower detection limit. CSW, commercial sex worker; HESN, HIV-1-exposed seronegative; IL, interleukin; IP-10, interferon- $\gamma$-induced protein 10;

MIG, monokine induced by interferon- $\gamma .{ }^{*} P<0.05$.

There was no correlation between IL-1 $\alpha$ and antiprotease expression observed that was specific only to the HESN group (Figure 5, Table 2, and Supplementary Table S4 online). Second, we observed fewer correlations overall between the antiprotease and the cytokines/chemokines in the HESN group compared with the other groups. Because of the large number of comparisons in this analysis, correlations with a more stringent $P$-value of $<0.005$ were considered significant. The HESN group showed only two significant correlations: between serpin A3 and MCP-1 $(P=0.0006)$ and between serpin A1 and IL-1 $\alpha$ $(P=0.0002)$. In the HIV-1-negative group, seven correlations were observed. In the HIV-1-infected group, Serpin A1 and A3 

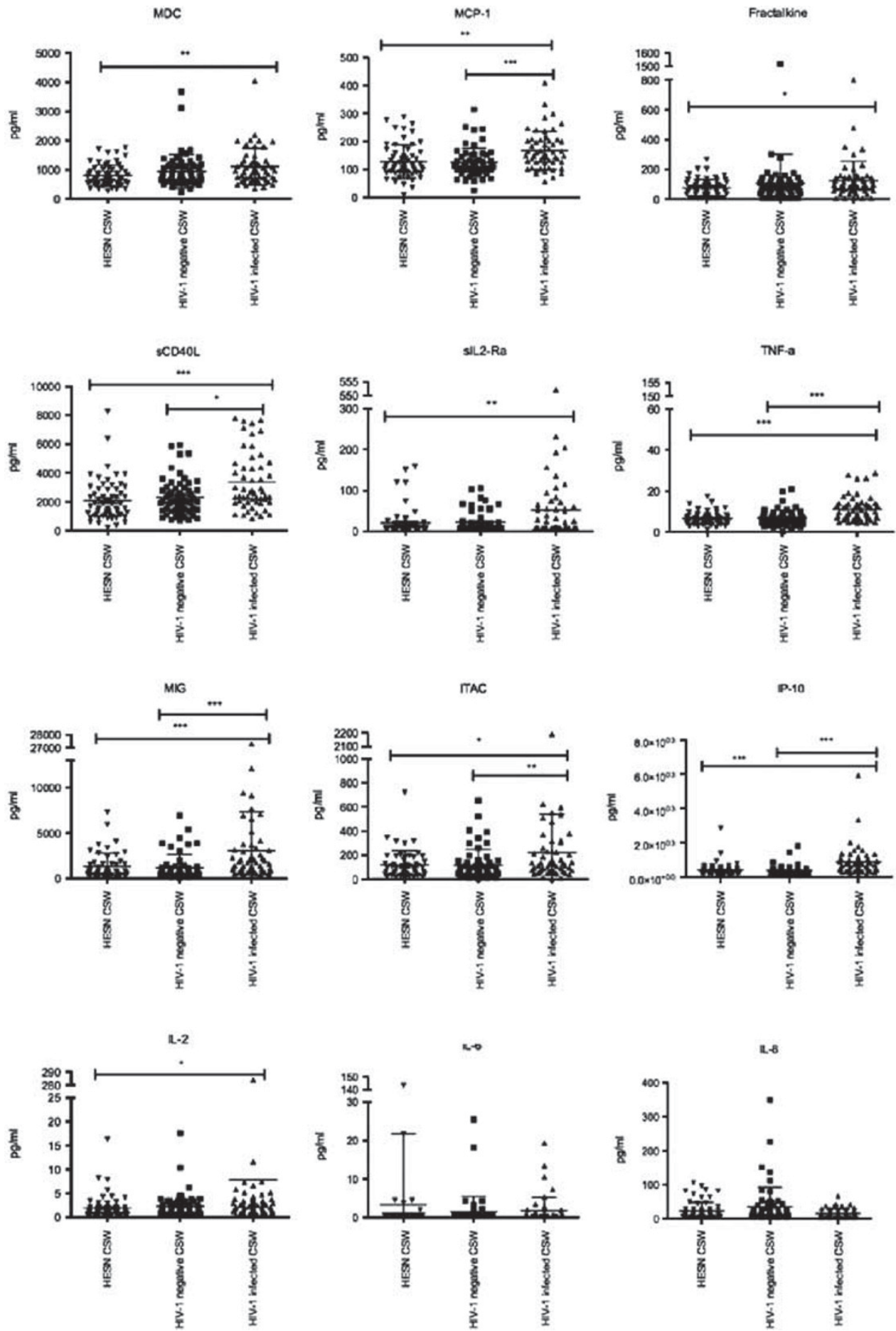

Figure 2 Distribution of significant association observed in the plasma. Systemic cytokine and chemokine levels were analyzed with Milliplex MAP multiplex and normalized with standard curves. Sample measurements below the lower detection limit were assigned a value of half the lower detection limit. ${ }^{*} P<0.05 ;{ }^{* *} P<0.01 ;{ }^{* *} P<0.0001$. CSW, commercial sex worker; HESN, HIV-1-exposed seronegative; IL, interleukin; IP-10, interferon$\gamma$-induced protein 10; I-TAC, interferon-inducible T-cell $\alpha$-chemoattractant; MCP-1, monocyte chemotactic protein 1; MDC, macrophage-derived chemokine; MIG, monokine induced by interferon- $\gamma$; TNF- $\alpha$, tumor necrosis factor- $\alpha$.

each correlated with 6 and 10 cytokines/chemokines, respectively (Table 2). Overall, fewer correlations were observed in the HESN compared with the other groups for the majority of the antiprotease tested. The only exception to this pattern is cystatin A, which did not correlate with cytokines/chemokines in the HIV-1 negative and HIV-1-infected CSWs but correlated with MIG and IL-1r $\alpha$ in the HESN group $(P=0.004$ and $P=0.0002$, respectively). 
CVL

Plasma
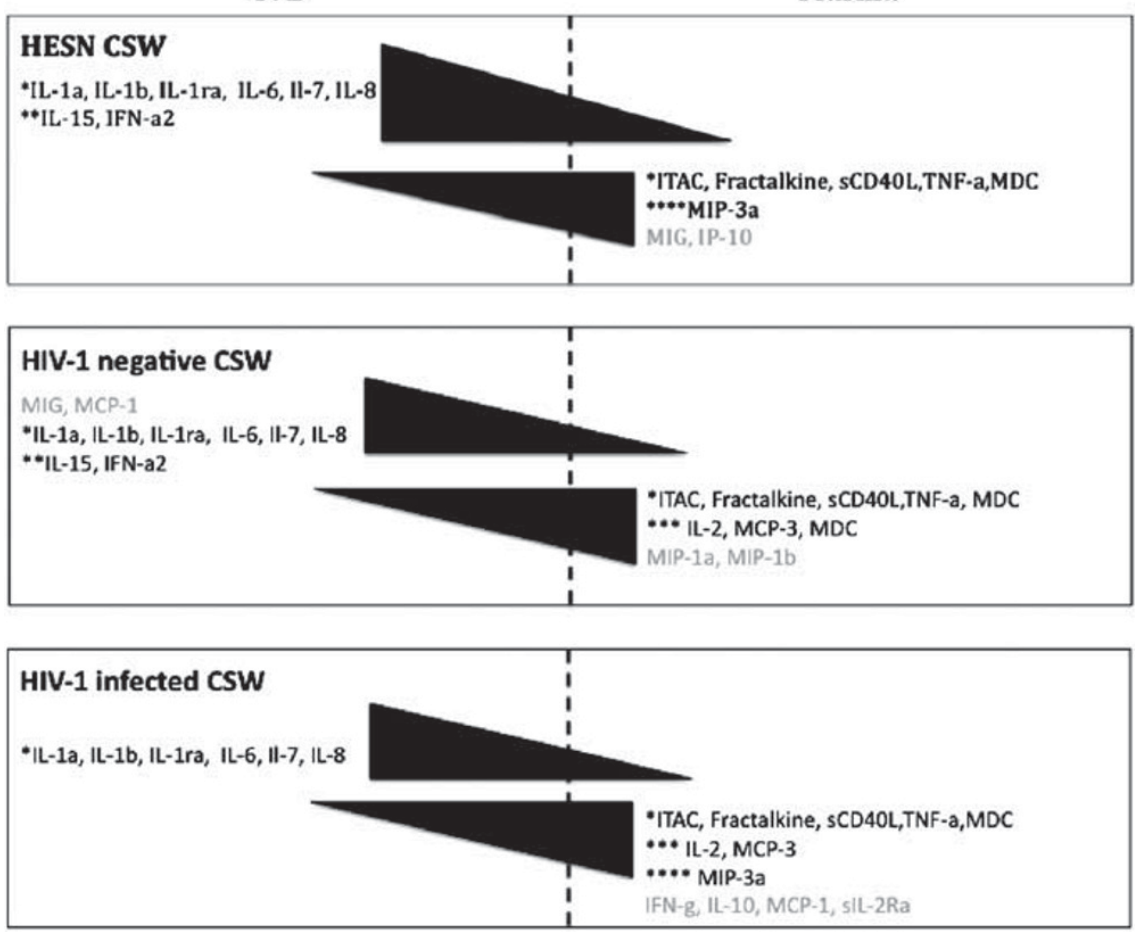

Figure 3 Schema of the gradient of expression between the mucosal and systemic compartments. *Same in the three groups. ${ }^{* \star}$ Similar between HIV-1-negative CSW and HESN CSW. ${ }^{* * *}$ Similar between HIV-1-negative CSW and HIV-1-infected CSW. ${ }^{* * *}$ Similar between HIV-1-infected CSW and HESN. CSW, commercial sex worker; CVL, cervicovaginal lavage; HESN, HIV-1-exposed seronegative; IFN, interferon; IL, interleukin; IP-10, interferon- $\gamma$-induced protein 10; I-TAC, interferon-inducible T-cell $\alpha$-chemoattractant; MCP, monocyte chemotactic protein; MDC, macrophage-derived chemokine; MIG, monokine induced by interferon- $\gamma$; MIP, macrophage inflammatory protein; TNF- $\alpha$, tumor necrosis factor- $\alpha$.

\section{DISCUSSION}

A better understanding of the mucosal immunology in the FGT and the correlates of HIV-1 protection in this compartment is needed if we are to develop effective microbicides and preventive strategies. This study showed that HESN individuals from the Pumwani Commercial Sex Worker Cohort from Nairobi, Kenya, exhibit a particular chemokine/cytokine expression pattern in the FGT that does not broadly correlates with antiprotease expression and might, in part, contribute to their resistance to HIV-1 infection. We also showed, confirming previous studies, ${ }^{11,29}$ that the mucosal and systemic compartments demonstrate different patterns of chemokine/cytokine expression and the study of one compartment cannot predict what will be observed at the other.

In this cohort, HESN have a lower genital tract expression of IL-1 $\alpha$, MIG, and IP-10 than HIV-1-negative or HIV-1-positive groups. These results are supported by a previous study of CSWs in Benin that showed a lower level of MIG in the CVL of HIV-1-uninfected CSWs compared with HIV-1-infected $\mathrm{CSWs},{ }^{29}$ demonstrating the potential importance of this chemokine in the resistance to HIV-1 infection.

MIG and IP-10 are two chemokines belonging to the IFN$\gamma$-inducible family. They both bind the G-protein-coupled receptor CXCR3, which is preferentially expressed by activated (mainly T helper type 1 ) and memory T cells, natural killer cells, and plasmocytoid dendritic cells. ${ }^{30,31}$ These two chemokines play an important role in leukocyte trafficking through their ability to promote the migration of natural killer and activated and memory, but not naive T cells. ${ }^{32,33}$ Recent studies showed that increased levels of MIG and IP-10 mRNA in the lymph node were positively associated with disease progression, and high blood levels of IP-10 could predict rapid disease progression. ${ }^{34}$ Furthermore, Stacey et al..$^{35}$ observed that IP-10 is one of the first chemokines to be increased in the plasma in the first days after HIV infection and remains elevated throughout infection. It is, therefore, quite striking that these two chemokines, which seem to be important in the establishment and progression of HIV infection, were lower in the CVL of the HESN group. Given their potential role in promoting disease progression, and perhaps the establishment of mucosal infection, their low levels of expression among HESN women suggests a potential role in protection from infection. It could be hypothesized that low basal levels of IP-10 and MIG fail to promote an immune environment that facilitates HIV infection by decreasing the recruitment of activated $\mathrm{T}$ cells at the port of entry of the virus.

Interestingly, production of both MIG and IP-10 is induced by the expression of IFN- $\gamma$, and polymorphisms in the IFN- $\gamma$-regulating gene $I R F 1$ have been associated with HIV protection in this cohort. ${ }^{21,36}$ Furthermore, in a cohort of HESN men who have sex with men, Hladik et al. ${ }^{37}$ showed that those HESN men had a lack of HIV-1-specific IFN- $\gamma$-secreting 


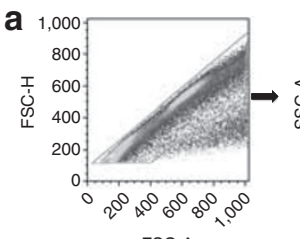

FSC-A

b
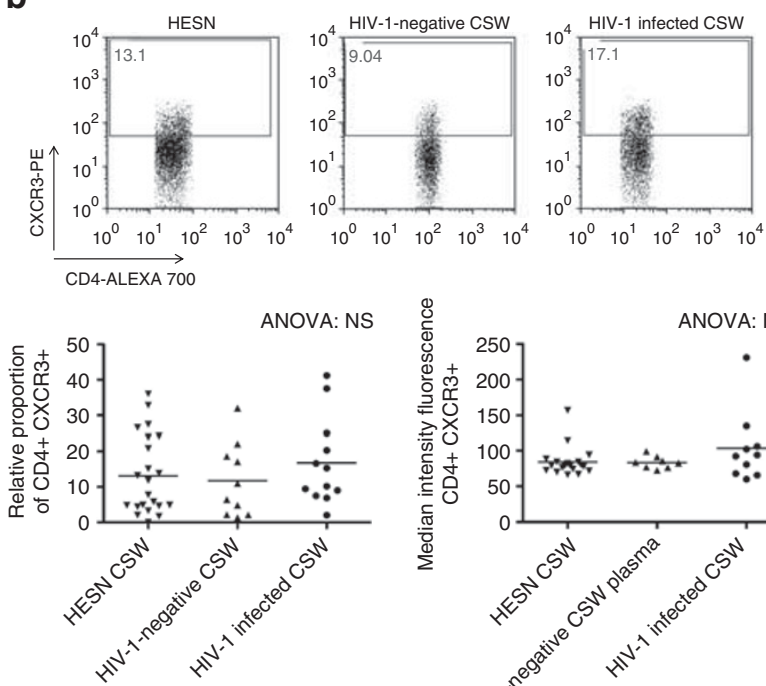

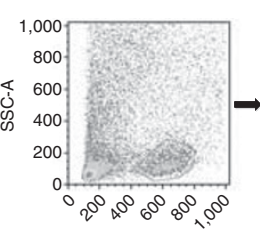

FSC-A

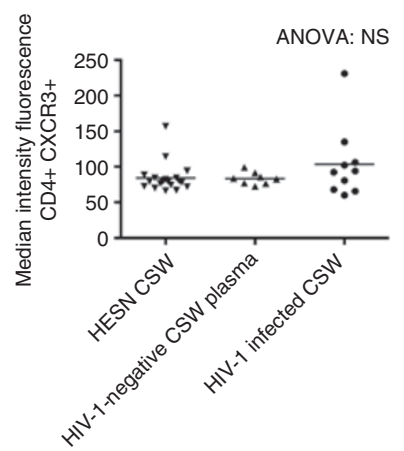

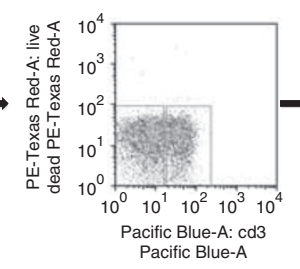
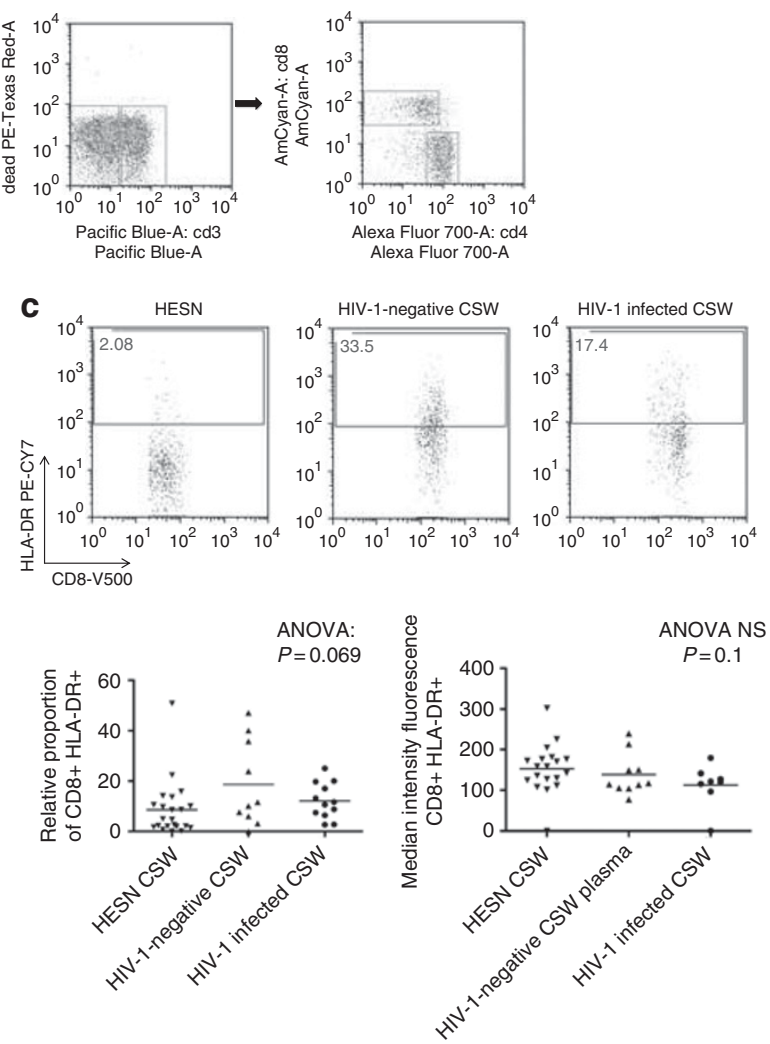

Figure 4 Relative percentage of T-cell subpopulations among cervicovaginal cells (cervical mononuclear cells (CMC)). (a) Gating strategy on singlet (first at the left), lymphocyte population, CD3 + cells alive, and CD4 and CD8+ T cells. (b) Relative percentage of CXCR3+ CD4+ T cells. (c) Relative percentage of HLA-DR + CD8 + T cells in HIV-1-negative, HIV-1-infected, and HESN CSWs. Cell population percentages were evaluated with oneway analysis of variance (ANOVA). CSW, commercial sex worker; FSC, forward scatter; HESN, HIV-1-exposed seronegative; HLA, human leukocyte antigen; NS, nonsignificant; SSC, side scatter.

T cells. Within the Pumwani cohort, we have also demonstrated that HIV-specific CD4 + T cells are characterized by robust proliferation but lower levels of IFN- $\gamma$ production. ${ }^{38}$ This study combined with those results shows the importance of the IFN- $\gamma$ pathway in the protection from HIV-1 infection.

Lower levels of IL- $1 \alpha$, MIG, and IP-10 at the mucosal environment may contribute to a phenotype of T-cell immune quiescence rather than of immune activation, which could result in fewer target cells available for the virus. ${ }^{22}$ This appears to be reflected by the observation of decreased HLA-DR expression on cervical CD8 + T cells among the HESN group. Furthermore, the fact that the proportion of cells expressing the receptor for MIG and IP-10, CXCR3, was the same in the three groups despite the lower levels of the two chemokines showed that at the mucosal site this lower chemokine level is not compensated by an increase in the expression of CXCR3. Recently, Li et al. ${ }^{39}$ showed, in monkeys, that specific inhibition of certain chemokines like MIP-3 $\alpha$ before infection was associated with vaginal protection to Simian immunodeficiency virus challenge, raising the possibility that lower MIG/IP-10 levels among HESN individuals may have a similar outcome.

By comparing the systemic and mucosal levels of MIG and IP-10, we observed a difference in the gradient of expression between groups. We observed that in the HESN individuals the level of MIG and IP-10 was lower in the CVL than in the blood, whereas in HIV-1-negative CSWs MIG was higher in the CVL. In HIV-1-negative individuals, the gradient of chemokines is trafficking the activated $\mathrm{T}$ cells to the FGT, which is the port of entry of the virus. This may explain why HIV-1-negative CSWs are at an increased risk of HIV-1 infection relative to the HESN group.

Many factors can influence chemokine/cytokine expression in the FGT. Antiproteases are innate proteins that have important functions in regulating inflammation and host defense. ${ }^{40,41}$ Serpin A1/A3, cystatin A/B, and A2ML1 are overexpressed among HESN in Pumwani cohort. ${ }^{28}$ Given the fact that antiprotease can influence cytokine/chemokine expression, matched samples from the Burgener et al. ${ }^{28}$ study were tested for cytokine/chemokine levels in this study. Not only are MIG, IP-10, and IL-1 $\alpha$ expressed at lower levels in the HESN group, but they are also differentially correlated with antiprotease expression compared with the other groups. This suggests that these cytokines/chemokines are uniquely regulated among the HESN group, Interestingly, the only antiprotease that correlated with both MIG and IP-10 (A2ML1) also correlated with IFN- $\gamma$, the driver of MIG and IP-10 expression. A limitation of this study may be the $P<0.005$ cutoff 

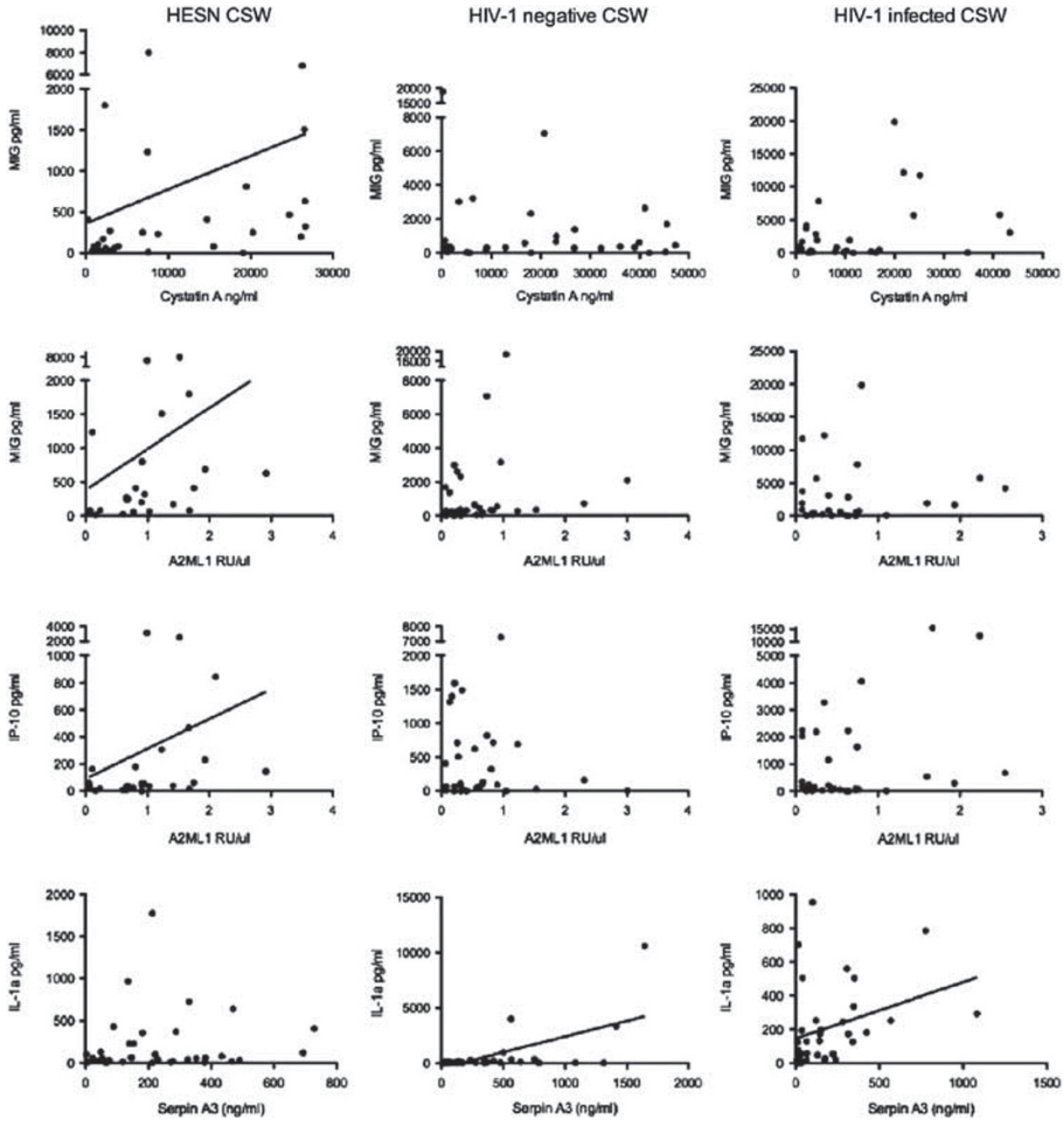

Figure 5 Correlations between MIG, IP-10, and IL-1 $\alpha$ and antiproteases in HIV-1-negative CSW, HIV-1-infected CSW, and HESN CSW. The $r$ value for: MIG vs. Cystatin A: 0.1 (HIV-1-negative CSW), 0.2 (HIV-1-infected CSW), 0.5 (HESN); MIG vs. A2ML1: 0.3 (HIV-1-negative CSW), 0.1 (HIV-1-infected CSW), 0.5 (HESN); IP-10 vs. A2ML1: - 0.01 (HIV-1-negative CSW), 0.2 (HIV-1-infected CSW), 0.5 (HESN); IL-A vs. serpin A3: 0.5 (HIV-1-negative CSW); 0.5 (HIV-1-infected CSW), and 0.1 (HESN). CSW, commercial sex worker; HESN, HIV-1-exposed seronegative; IL, interleukin; IP-10, interferon- $\gamma$-induced protein 10; MIG, monokine induced by interferon- $\gamma$.

used in the proteomic analysis that may have introduced a type $1 r$ error (which is introduction of a false positive). However, $P<0.005$ was chosen as a balance between increasing the stringency of the analysis by 10 -fold and, given the discovery, nature of the study, avoiding a type $2 r$ error, which is missing a real finding.

Together, these data suggest a model of the HESN FGT environment where antiproteases are increased and inflammatory cytokine IL- $1 \alpha$ and chemoattractants MIG and IP-10 are decreased independently of each other. We speculate that this could result in a synergistic effect that could decrease the abundance of activated target cells for propagation of infection, thereby reducing the likelihood of HIV transmission through the mucosal barrier. Unfortunately, this study does not allow us to determine the drivers that lead to relative expression differences of MIG, IP-10, and IL- $1 \alpha$. Further studies are required to determine whether genetic polymorphisms, relative cell abundance, and/or cytokine production capacity contribute to the observed expression levels. The causal factors governing the relationship between antiprotease and cytokine expression are, if any, also currently unknown and require investigation.

In conclusion, in this study, we showed that HIV-1-resistant women have a distinct pattern of mucosal chemokine/cytokine expression that contributes to the immune quiescence phenotype observed in the Pumwani HESN cohort. HESN subjects showed specific mucosal characteristics (lower levels of IL- $1 \alpha$, MIG, and IP-10 correlated with higher level of antiproteases) that may be critical at the first lines of defense during exposure to HIV-1 infection. HESN subjects seem to have a better control of mucosal T-cell inflammation, but at the same time are able to drive an innate antiviral response and prevent the spread of the infection to the systemic compartment. These data give us new important insights into mucosal immunity in 
Table 2 Correlation of cytokines/chemokines with antiproteases. (a) Significant correlation between MIG, IP-10, and IL-1 $\alpha$ and antiproteases $(P<0.05)$; (b) significant correlation between cytokines/chemokines and antiproteases after adjustment for multiple correlations $(P<0.005)$.

\begin{tabular}{|c|c|c|c|}
\hline & $\begin{array}{l}\text { HESN } \\
\text { CSW }\end{array}$ & $\begin{array}{l}\text { HIV-1-negative } \\
\text { CSW }\end{array}$ & $\begin{array}{l}\text { HIV-1-infected } \\
\text { CSW }\end{array}$ \\
\hline \multicolumn{4}{|l|}{ (a) } \\
\hline \multirow[t]{3}{*}{ MIG } & $\begin{array}{l}\text { Cystatin A } \\
(P=0.004)\end{array}$ & NS & NS \\
\hline & $\begin{array}{l}\text { A2ML1 } \\
(P=0.012)\end{array}$ & NS & NS \\
\hline & $\begin{array}{l}\text { Serpin B4 } \\
(P=0.011)\end{array}$ & NS & NS \\
\hline \multirow[t]{2}{*}{ IP-10 } & $\begin{array}{l}\text { A2ML1 } \\
(P=0.013)\end{array}$ & NS & $\begin{array}{l}\text { Serpin A1 } \\
(P=0.009)\end{array}$ \\
\hline & $\begin{array}{l}\text { Serpin A3 } \\
(P=0.049)\end{array}$ & NS & $\begin{array}{l}\text { Serpin A3 } \\
(P=0.0002)\end{array}$ \\
\hline \multirow[t]{3}{*}{$\mathrm{IL}-1 \alpha$} & $\begin{array}{l}\text { Serpin A1 } \\
(P=0.0002)\end{array}$ & $\begin{array}{l}\text { Serpin A1 } \\
(P=0.00003)\end{array}$ & $\begin{array}{l}\text { Serpin A1 } \\
(P=0.002)\end{array}$ \\
\hline & NS & $\begin{array}{l}\text { Serpin A3 } \\
(P=0.001)\end{array}$ & $\begin{array}{l}\text { Serpin A3 } \\
(P=0.005)\end{array}$ \\
\hline & NS & $\begin{array}{l}\text { Cystatin B } \\
(P=0.002)\end{array}$ & NS \\
\hline \multicolumn{4}{|l|}{ (b) } \\
\hline \multirow[t]{6}{*}{$\begin{array}{l}\text { Serpin } \\
\text { A3 }\end{array}$} & $\begin{array}{l}\text { MCP-1 } \\
(P=0.001)\end{array}$ & $\mathrm{IL}-1 \alpha(P=0.001)$ & $\mathrm{MIG}(P=0.001)$ \\
\hline & & $\mathrm{IL}-1 \beta(P=0.005)$ & $\mathrm{IP}-10(P=0.0002)$ \\
\hline & & & $\mathrm{IL}-1 \alpha(P=0.005)$ \\
\hline & & & IL-6 $(P=0.0004)$ \\
\hline & & & IL-8 $(P=0.00010$ \\
\hline & & & MCP-1 $(P=0.0010)$ \\
\hline \multirow[t]{10}{*}{$\begin{array}{l}\text { Serpin } \\
\text { A1 }\end{array}$} & $\begin{array}{l}\mathrm{IL}-1 \alpha \\
(P=0.0002)\end{array}$ & $\begin{array}{l}\mathrm{IL}-1 \alpha \\
(P=0.00003)\end{array}$ & $\begin{array}{l}\text { Fractalkine } \\
(P=0.0003)\end{array}$ \\
\hline & & $\begin{array}{l}\text { IL-1 } \beta \\
(P=0.00030\end{array}$ & $\mathrm{IL}-1 \alpha(P=0.002)$ \\
\hline & & $\begin{array}{l}\text { MIP-1 } \alpha \\
(P=0.003)\end{array}$ & $\mathrm{IL}-1 \beta\left(P=5.8^{\mathrm{e}}-8\right)$ \\
\hline & & & IL-6 $(P=0.001)$ \\
\hline & & & IL-8 $(P=2.1$ e-6) \\
\hline & & & IL-10 ( $P=0.005)$ \\
\hline & & & IL-17 (P=0.0005) \\
\hline & & & MIP $-1 \beta(P=0.006)$ \\
\hline & & & $\begin{array}{l}\text { MCP-3 } \\
(P=0.0004)\end{array}$ \\
\hline & & & $\begin{array}{l}\text { TNF- } \alpha \\
(P=0.00001)\end{array}$ \\
\hline \multirow[t]{2}{*}{ A2ML1 } & & IL-15 (P=0.003) & \\
\hline & & $\begin{array}{l}\text { SCD40L } \\
(P=0.005)\end{array}$ & \\
\hline Cystatin A & $\begin{array}{l}\mathrm{MIG} \\
(P=0.004)\end{array}$ & & \\
\hline Serpin B4 & $\begin{array}{l}\mathrm{IL}-1 \mathrm{R} \alpha \\
(P=0.007)\end{array}$ & & \\
\hline
\end{tabular}

CSW, commercial sex worker; HESN, HIV-1-exposed seronegative; IL, interleukin; IP-10, interferon- $\gamma$-induced protein 10; MCP, monocyte chemotactic protein; MIG, monokine induced by interferon- $\gamma$; MIP, macrophage inflammatory protein; $s$, soluble; TNF- $\alpha$, tumor necrosis factor- $\alpha$. order to develop effective preventive strategies and highlight the importance of the IFN- $\gamma$ pathway in resistance to HIV-1 infection.

\section{METHODS}

Study population. The Pumwani Sex Worker Cohort was established in 1985 for a study on the immunobiology and epidemiology of sexually transmitted infections. Over 3,000 women have been enrolled in this open cohort. In all, 57 HESN CSWs, 51 HIV-1-negative CSWs, and 67 HIV-1-infected CSWs were included in this study. HESN CSWs were defined as active CSWs who have remained HIV-1 seronegative and HIV-1 negative by PCR for at least 7 years of follow-up. HIV-1negative CSWs are women newly enrolled in the cohort (for $<3$ years) and HIV-1 seronegative. Each woman enrolled in the study answered a questionnaire for sociodemographics, sexual behavior information, duration of sex work, number of sex clients, condom use, number of regular partners, and reproductive history. This study was approved by the research ethics boards of both the University of Manitoba and University of Nairobi. Each woman underwent a gynecological exam. Vaginal specimens were obtained to test for the presence of bacterial vaginosis, Neisseria gonorrhoeae, and Chlamydia sp. Neisseria gonorrhoeae and Chlamydia sp were tested by PCR and presence of bacterial vaginosis was tested by Nugent score.

CVL sample collection. The endocervix was washed with $2 \mathrm{ml}$ of sterile $1 \times$ phosphate-buffered saline and the lavage was collected from the posterior fornix. Samples were placed into a $15 \mathrm{ml}$ conical tube, centrifuged to remove cellular debris, and the supernatant stored at $-70^{\circ} \mathrm{C}$ and shipped in liquid nitrogen dry shipper to Winnipeg, Manitoba.

Plasma sample collection. Peripheral blood was collected in EDTA tubes. Plasma were frozen at $-80^{\circ} \mathrm{C}$, and shipped by dry shipper to Canada where they were stored at $-70^{\circ} \mathrm{C}$ until used. Plasma and CVL samples were collected on the same day. When available, matched plasma, with CVL already tested, were analysed for chemokine and cytokine measurement.

Chemokine and cytokine measurement. Chemokine levels were determined using the Milliplex MAP multiplex kit (Human Cytokine/ Chemokine I, II from Millipore, Billerica, MA) and analyzed on the BioPlex-200 (Bio-Rad, Mississauga, ON, Canada). CVL samples were analyzed according to the overnight protocol given by the manufacturer, whereas plasma was analyzed according to the 2-h incubation protocol given by the manufacturer. Lower detection limit was $10.6 \mathrm{pg} \mathrm{ml}^{-1}$ for Fractalkine, $40.6 \mathrm{pg} \mathrm{ml}^{-1}$ for IFN- $\alpha 2,0.3 \mathrm{pg} \mathrm{ml}^{-1}$ for IFN- $\gamma$, IL- 8 , and IL$17,6.4 \mathrm{pg} \mathrm{ml}^{-1}$ for IL- $1 \alpha$ and MIP-1 $\alpha, 0.7 \mathrm{pg} \mathrm{m}^{-1}$ for IL- $1 \beta, \mathrm{IL}-6$, and IL-15, $5.5 \mathrm{pg} \mathrm{ml}^{-1}$ for IL-1 $\mathrm{r} \alpha, 0.6 \mathrm{pg} \mathrm{ml}^{-1}$ for IL-2, $0.5 \mathrm{pg} \mathrm{ml}^{-1}$ for IL-10, $2.2 \mathrm{pg} \mathrm{ml}^{-1}$ for IP-10, $1.6 \mathrm{pg} \mathrm{ml}^{-1}$ for MCP-1, 3.7 $\mathrm{pg} \mathrm{ml}^{-1}$ for MCP-3, 6.9 for macrophage-derived chemokine, $8.9 \mathrm{pg} \mathrm{ml}^{-1}$ for MIP-1 $\beta, 7.7 \mathrm{pg} \mathrm{ml}^{-1}$ for sIL-2R $\alpha, 9.0 \mathrm{pg} \mathrm{ml}^{-1}$ for sCD40L, $19.4 \mathrm{pg} \mathrm{m}^{-1}$ for MIG, 2.9 for MIP$3 \alpha, 0.8$ for I-TAC, and $0.1 \mathrm{pg} \mathrm{ml}^{-1}$ for tumor necrosis factor- $\alpha$. Samples below the lower detection limit were assigned a value of half of the lower detection limit in $\mathrm{pg} \mathrm{ml}^{-1}$.

Evaluation of CMC population by flow cytometry. CMCs were collected using both a cytobrush and scraper. CMCs were obtained from 12 HIV-1-infected CSWs, 10 HIV-1-negative CSWs, and 32 HESN CSWs. Briefly, CMCs were collected from all women under speculum examination by inserting the cytobrush and the scraper into the endocervical os, rotating $360^{\circ}$, and immediately placing in $5 \mathrm{ml}$ of phosphate-buffered saline. Cytobrush samples with visible blood contamination were excluded for further analysis. Samples were kept on ice and transported from the Pumwani clinic to the laboratory. Upon arrival in the laboratory, the cytobrushes and scraper were vortexed and cells were flushed out of the brush. Two washes were performed (first with $5 \mathrm{ml}$ of RPMI and second with $5 \mathrm{ml}$ of phosphate-buffered saline) each followed by a 10-min centrifugation. CMCs were freshly labeled with a cocktail containing the 
following mouse anti-human monoclonal antibodies: fluorescein isothiocyanate-conjugated anti-CCR5; phycoerythrin (PE) anti-CXCR3; PEcyanine (Cy)7-conjugated anti-HLA-DR; PE-Cy5-conjugated anti-CD69, allophycocyanin-conjugated anti-CD56; allophycocuanin $\mathrm{H} 7$ conjugated anti-CD16; Alexa fluor 700-conjugated anti-CD4; V500 anti-CD8 and V450 anti-CD3 (Becton Dickinson (BD) Biosciences, Mississauga, ON, Canada). Dead cells were identified with live-dead red (PE-Texas red; Invitrogen, Burlington, ON, Canada). Data acquisition of all events per sample was performed on a BD LSLR II apparatus (Becton Dickinson), and the analysis was done using FlowJO software (version 9.3.1; Tree Star, Ashland, OR).

Measurement of antiprotease concentrations in CVL samples. Antiproteases were quantified using a combination of enzymelinked immunosorbent assay (ELISA) and semiquantitative western blot. Western immunoblotting of A2ML1/Serpin B4: Protein $(1 \mu \mathrm{g})$ from each CVL sample was resolved by sodium dodecyl sulfate polyacrylamide gel electrophoresis (SDS-PAGE) gels (Invitrogen) and transferred to nitrocellulose membranes using the iBlot transfer system (Invitrogen). Bands were developed with Immobilon detection reagent (Millipore), and volumetric analysis performed using Quantity One software (Bio-Rad). ELISA assays of Cystatin A/B, Serpin A1/A3: A sandwich ELISA was developed to quantify Cystatin B, and Serpin A1/Serpin A3 were measured using commercially available human A1AT and Alpha 1-Antichymotrypsin ELISA kits (Immunology Consultants Laboratory, Newberg, OR), according to the manufacturer's protocol. All samples were run in duplicate and blinded to the study group of which they belonged. See Burgener et al. ${ }^{28}$ for more detailed protocols.

Statistical analyses. Analyses were performed using GraphPad Prism (version 5.0, GraphPad Software, La Jolla, CA). The $\chi^{2}$ test was used to assess the significance of the associations between categorical variable, Gaussian distribution was test by D'agostino and Pearson's omnibus normality test and Shapiro-Wilk normality test. One-way analysis of variance (Kruskal-Wallis test) and Mann-Whitney $U$-test were used for variables that were not normally distributed between three groups and two groups, respectively. Spearman's rank test was used to determine correlations between continuous variables. Wilcoxon matched-pairs test was used to compare cytokine/chemokine expression in the CVL and plasma samples. For proteomics, because multiples correlations were performed, the $P$-value threshold for significance was reduced to $P=0.005$. This value was predetermined as a balance between the need to increase stringency due to the multiple comparisons and, given the discovery approach of this study, not making a type 2 error, which is missing a real effect.

SUPPLEMENTARY MATERIAL is linked to the online version of the paper at http://www.nature.com/mi

\section{ACKNOWLEDGMENTS}

Julie Lajoie holds a postdoctoral research award from the CIHR and is a trainee in the CIHR international infectious disease and global health training program (IID\&GHTP). Jennifer Juno is funded by the IID\&GHTP. Keith Fowke holds a Manitoba Research Chair from the Manitoba Health Research Council. This study was funded by the CIHR operating Grant MOP-86721. We appreciate the technical support of S. Ramdahin and C. Mesa. We thank Paul McLaren for help in performing principal component analysis. We are grateful to all the staff and clients of the Pumwani clinic and technicians at the Kenya AIDS Control Program at the University of Nairobi.

\section{DISCLOSURE}

The authors declared no conflict of interest.

2012 Society for Mucosal Immunology

\section{REFERENCES}

1. Rapport de l'épidémie mondiale du SIDA 2010.

2. Abdool, K.Q. et al. Effectiveness and safety of tenofovir gel, an antiretroviral microbicide, for the prevention of HIV infection in women. Science 329, 1168-1174 (2010).

3. McCormack, S. et al. PRO2000 vaginal gel for prevention of HIV infection (microbicides development programme 301); a phase 3, randomized, double-blind, parallel-group trial. Lancet 376, 1329-1337 (2010).

4. Prohaska, T.R., Albrecht, G., Levy, J.A., Sugrue, N. \& Kim, J.H. Determinants of self-perceived risk for AIDS. J. Health Soc. Behav. 4, 384-394 (1990).

5. Ahlburg, D.A., Jensen, E.R. \& Perez, A.E. Determinants of extramarital sex in the Philippines. Health Transit Rev. 7 (Suppl), 467-479 (1997).

6. McClelland, R.S., Lavreys, L., Hassan, W.M., Mandaliya, K., NdinyaAchola, J.O. \& Baeten, J.M. Vaginal washing and increased risk of HIV-1 acquisition among African women: a 10-year prospective study. AIDS 20, 269-273 (2006).

7. Lajoie, J., Hargrove, J., Zijenah, L.S., Humphrey, J.H., Ward, B.J. \& Roger, M. Genetic variants in nonclassical major histocompatibility complex class I human leukocyte antigen (HLA)-E and HLA-G molecules are associated with susceptibility to heterosexual acquisition of HIV-1. J. Infect. Dis. 193, 298-301 (2006).

8. Kaslow, R.A. et al. Influence of combinations of human major histocompatibility complex genes on the course of HIV-1 infection. Nat. Med. 4, 405-411 (1996).

9. Young, J.M., Turpin, J.A., Musib, R. \& Sharma, O.K. Outcomes of a National Institute of Allergy and Infectious Diseases Workshop on understanding HIV-exposed but seronegative individuals. AIDS Res. Hum. Retroviruses 7, 737-743 (2011).

10. Fowke, K.R. et al. Resistance to HIV-1 infection among persistently seronegative prostitutes in Nairobi, Kenya. Lancet 348, 1347-1351 (1996).

11. Lajoie, J. et al. Differences in immunoregulatory cytokine expression patterns in the systemic and genital tract compartments of HIV-1-infected commercial sex workers in Benin. Mucosal Immunol. 4, 309-316 (2008).

12. Jennes, W. et al. Cutting edge: resistance to HIV-1 infection among African female sex workers is associated with inhibitory KIR in the absence of their HLA ligands. J. Immunol. 10, 6588-6592 (2006).

13. Clerici, M. et al. Cell-mediated immune response to human immunodeficiency virus (HIV) type 1 in seronegative homosexual men with recent sexual exposure to HIV-1. J. Infect. Dis. 6, 1012-1019 (1992).

14. Mazzoli, S. et al. HIV-specific mucosal and cellular immunity in HIVseronegative partners of HIV-seropositive individuals. Nat. Med. 11, 1250-1257 (1997).

15. Ballan, W.M. et al. Natural killer cells in perinatally HIV-1-infected children exhibit less degranulation compared to HIV-1-exposed uninfected children and their expression of KIR2DL3, NKG2C, and NKp46 correlates with disease severity. J. Immunol. 179, 3362-3370 (2007).

16. Tomescu, C., Abdulhaqq, S. \& Montaner, L.J. Evidence for the innate immune response as a correlate of protection in human immunodeficiency virus (HIV)-1 highly exposed seronegative subjects (HESN). Clin. Exp. Immunol. 164, 158-169 (2011).

17. Kaul, R. et al. New insights into HIV-1 specific cytotoxic T-lymphocyte responses in exposed, persistently seronegative Kenyan sex workers. Immunol. Lett. 79, 3-13 (2001).

18. Kaul, R. et al. HIV-1-specific mucosal CD8+ lymphocyte responses in the cervix of HIV-1-resistant prostitutes in Nairobi. J. Immunol. 164, 1602-1611 (2000).

19. Rowland-Jones, S.L. et al. How important is the 'quality' of the cytotoxic T lymphocyte (CTL) response in protection against HIV infection? Immunol. Lett. 79, 15-20 (2001).

20. Burgener, A. et al. Identification of differentially expressed proteins in the cervical mucosa of HIV-1-resistant sex workers. J. Proteome Res. 7, 4446-4454 (2008).

21. Ji, H., Ball, TB., Ao, Z., Kimani, J., Yao, X. \& Plummer, F.A. Reduced HIV-1 long terminal repeat transcription in subjects with protective interferon regulatory factor-1 genotype: a potential mechanism mediating resistance to infection by HIV-1. Scand. J. Infect. Dis. 42, 389-394 (2010).

22. McLaren, P.J. et al. HIV-exposed seronegative commercial sex workers show a quiescent phenotype in the CD4+ T cell compartment and 
reduced expression of HIV-dependent host factors. J. Infect. Dis. 202 (Suppl 3), S339-S344 (2010).

23. Narimatsu, R., Wolday, D. \& Patterson, B.K. IL-8 increases transmission of HIV type 1 in cervical explant tissue. AIDS Res. Hum. Retroviruses 21 228-233 (2005).

24. Iqbal, S.M. et al. Elevated T cell counts and RANTES expression in the genital mucosa of HIV-1-resistant Kenyan commercial sex workers. J. Infect. Dis. 192, 728-738 (2005).

25. Shin, L.Y. \& Kaul, R. Stay it with flora: maintaining vaginal health as a possible avenue for prevention of human immunodeficiency virus acquisition. J. Infect. Dis. 197, 1355-1357 (2008).

26. Hirbod, T. et al. Upregulation of interferon-alpha and RANTES in the cervix of HIV-1-seronegative women with high-risk behavior. J. Acquir. Immune. Defic. Syndr. 43, 137-143 (2006).

27. Campbell, G.R. \& Spector, S.A. CCL2 increases X4-tropic HIV-1 entry into resting CD4+ T cells. J. Biol. Chem. 283, 30745-30753 (2008).

28. Burgener, A. et al. A comprehensive proteomic study identifies serpin and cystatin antiproteases as novel correlates of HIV-1 resistance in the cervicovaginal mucosa of female sex workers. J. Proteome. Res. 10, 5139-5149 (2011).

29. Lajoie, J. et al. Chemokine expression patterns in the systemic and genital tract compartments are associated with HIV-1 infection in women from Benin. J. Clin. Immunol. 30, 90-98 (2010).

30. Thapa, M. \& Carr, D.J. CXCR3 deficiency increases susceptibility to genital herpes simplex virus type 2 infection: uncoupling of CD8+ T-cell effector function but not migration. J. Virol. 83, 9486-9501 (2009).

31. Hsieh, M.F. et al. Both CXCR3 and CXCL10/IFN-inducible protein 10 are required for resistance to primary infection by dengue virus. J. Immunol. 177, 1855-1863 (2006).

32. Farber, J.M. Mig and IP-10: CXC chemokines that target lymphocytes. J. Leukoc. Biol. 61, 246-257 (1997).
33. Muller, M., Carter, S., Hofer, M.J. \& Campbell, I.L. Review: the chemokine receptor CXCR3 and its ligands CXCL9, CXCL10 and CXCL11 in neuroimmunity--a tale of conflict and conundrum. Neuropathol. Appl. Neurobiol. 36, 368-387 (2010).

34. Durudas, A., Milush, J.M., Chen, H.L., Engram, J.C., Silvestri, G. \& Sodora, D.L. Elevated levels of innate immune modulators in lymph nodes and blood are associated with more-rapid disease progression in simian immunodeficiency virus-infected monkeys. J. Virol. 83, 12229-12240 (2009).

35. Stacey, A.R. et al. Induction of a striking systemic cytokine cascade prior to peak viremia in acute human immunodeficiency virus type 1 infection, in contrast to more modest and delayed responses in acute hepatitis B and C virus infections. J. Virol. 83, 3719-3733 (2009).

36. Ball, T.B. et al. Polymorphisms in IRF-1 associated with resistance to HIV-1 infection in highly exposed uninfected Kenyan sex workers. AIDS 21, 1091-1101 (2007).

37. Hladik, F. et al. Most highly exposed seronegative men lack HIV-1specific, IFN-gamma-secreting T cells. J. Immunol. 171, 2671-2683 (2003).

38. Alimonti, J.B. et al. CD4+ T cells responses in HIV-exposed seronegative women are qualitatively distinct from those in $\mathrm{HIV}$ infected women. J. Invest. Dis. 191, 20-24 (2005).

39. Li, Q. et al. Glycerol monolaurate prevents mucosal SIV transmission. Nature 458, 1034-1038 (2009).

40. Mangan, M.S., Kaiserman, D. \& Bird, P.I. The role of serpins in vertebrate immunity. Tissue Antigens. 72, 1-10 (2008).

41. Silverman, G.A. et al. The serpins are an expanding superfamily of structurally similar but functionally diverse proteins. Evolution, mechanism of inhibition, novel functions, and a revised nomenclature. J. Biol. Chem. 276, 33293-33296 (2001). 